\title{
Norois
}

Environnement, aménagement, société

206 | 2008/1

Itinéraires de lieux touristiques littoraux

\section{Les commmunautés vacancières}

Définir un nouveau type de lieux touristiques à partir de la Côte d'Albâtre (Seine-Maritime)

Holiday communities. Definition of a new type of place for tourism out of the Côte d'Albâtre case study (Normandy)

\section{Philippe Duhamel}

\section{(2) OpenEdition}

\section{Journals}

Édition électronique

URL : https://journals.openedition.org/norois/241

DOI : 10.4000/norois.241

ISBN : 978-2-7535-1554-3

ISSN : 1760-8546

Éditeur

Presses universitaires de Rennes

\section{Édition imprimée}

Date de publication : 1 mars 2008

Pagination : 21-36

ISBN : 978-2-7535-0690-9

ISSN : 0029-182X

\section{Référence électronique}

Philippe Duhamel, «Les commmunautés vacancières », Norois [En ligne], 206 | 2008/1, mis en ligne le 01 mars 2010, consulté le 14 janvier 2022. URL : http://journals.openedition.org/norois/241 ; DOI :

https://doi.org/10.4000/norois.241 


\title{
LES COMMMUNAUTÉS VACANCIÈRES
}

\section{DÉFINIR UN NOUVEAU TYPE DE LIEUX TOURISTIQUES}

À PARTir de la Côte d’Albâtre (SEIne-Maritime)

\author{
Philippe Duhamel \\ EA-MIT \\ (Université Paris 7 - Denis-Diderot), \\ Institut de géographie, 191 rue Saint-Jacques - 75005 Paris \\ ph.duhamel@wanadoo.fr
}

\section{RÉSUMÉ}

un travail de réflexion a été entrepris depuis près de dix ans par l'Équipe MIT et a permis de produire une typologie des lieux touristiques. Cet article vise à compléter ce dispositif en proposant un nouveau type: "les communautés vacancières » qui seraient une forme dérivée de la station, une station qui aurait évolué vers un lieu presque exclusivement réservé aux résidents secondaires, sans hébergement banalisé ou presque. Notre objectif est donc de définir cette catégorie de lieux grâce à une lecture historique des lieux touristiques de la Côte d'Albâtre et par l'observation de leur situation actuelle. Ainsi pourront être mieux compris les processus qui mènent à ce type de lieux. Sans répondre à toutes les questions, notre travail posera un certain nombre de jalons.

Mots-CLÉS : Côte d'Albâtre - Évolution des lieux - Histoire du tourisme - Résidents secondaires - Station - Ville touristique.

\section{ABSTRACT}

\section{Holiday communities. Definition of a new type of place for tourism out of the Côte d'Albâtre case study (Normandy)}

Researches have been undertaken for almost ten years by the MIT team and have led to the creation of a typology of tourist places. This article aims to complete this iventory by suggesting a new type "holiday communities" which would be a derived form of the station, a station which would have evolved to become a place almost reserved exclusively for second home owners residents, without hardly any accommodation. Our objective is to define this category of places by analysing the history of tourism on Côte d'Albatre and by observing the present situation. This will lead to a clearer understanding of the process which resulted in the creation this kind of places. This work will not claim to answer all the questions of the subject but will pave the way for future researches.

KEYWORDS : Côte d'Albâtre - History of tourism - Places development - Second home owners residents - Station - Tourist city. 


\section{Mise en place d'une problématique}

Depuis de nombreuses années, nous participons à un travail collectif de formalisation d'une typologie des lieux touristiques (Knafou et al., 1997, Équipe MIT, 2002 et Stock [coord.], 2003). Régulièrement, nous sommes confrontés à des difficultés face à des situations spatiales que nous ne parvenons pas à qualifier. De quoi s'agit-il ici ?

De nombreux lieux touristiques, tout en ayant «l'allure » de stations, ne semblent pas en être tout à fait. Certes une réelle animation existe par la fréquentation qui s'y observe dans les rues comme sur la plage pendant la saison estivale mais la population touristique semble avant tout constituée de fidèles, habitués du lieu, propriétaires de résidences secondaires. Ainsi les hébergements marchands (hôtels, camping, gîte...) seraient absents ou presque inexistants.

Longtemps a été évoquée l'idée de « lieux de vacances » pour les différencier des « lieux touristiques » tels que nous les définissons habituellement. Mais cela n'était que partiellement satisfaisant car le terme très générique de «vacances » pouvait créer des confusions. Progressivement, l'appréciation de la situation s'affinait par un heureux parallèle opéré entre ces « lieux de vacances » fonctionnant selon l'idée d'une population de fidèles qui revenaient dans un lieu investi par leur famille depuis une ou plusieurs générations et les gated communities.

Ici point de clôture matérielle mais des ressorts voisins dans le fonctionnement : l'association d'un groupe de personnes ayant en commun un projet de vie dans un lieu précis, à eux seuls « accessible » ou ceux qu'ils désignent, créant une dissociation entre les insiders et les outsiders. De rares extérieurs peuvent y séjourner grâce à la présence de quelques rares hébergements marchands car les résidences secondaires ne sont généralement pas proposées à la location et le lieu ne présente pas les attraits nécessaires à la venue d'un grand nombre de touristes.

De tout cela naquit une appellation au croisement de lieux de vacances et de communautés, les « communautés vacancières ».

\section{Des observations à valider}

Mais le mot ne suffit pas à valider la notion, encore faut-il la caractériser, la formaliser et valider les premières hypothèses avancées. C'est l'objet du présent article, première étape vers une compréhension de ce que revêt précisément la communauté vacancière. Loin de résoudre la question, nous nous proposons de poser quelques jalons à travers deux hypothèses.

- La communauté vacancière concernerait des espaces anciennement mis en tourisme où la présence du marché touristique aurait été réduite dans le passé et le serait toujours : aujourd'hui, pas de Tour opérateur pour proposer la destination, qu'il soit international ou national et peu hébergements marchands. Mais cela n'exclut pas d'autres formes d'hébergements touristiques, massives parfois, comme les résidences secondaires qui seraient, en l'occurrence, le fer de lance de l'accueil touristique.

- La deuxième hypothèse serait de savoir si les communautés vacancières sont des stations touristiques qui se sont progressivement orientées vers l'accueil des seuls résidents secondaires ou des lieux qui n'ont jamais vraiment été des stations ou alors des stations de petites tailles favorisant un entre-soi extrêmement poussé (Équipe MIT, 2002), et sorties du marché touristique peu de temps après leur développement touristique.

\section{Un espace pour la réflexion}

Pour appliquer cette réflexion à un premier terrain, nous avons choisi la Côte d'Albâtre au sens large de l'appellation. D'Étretat à Ault (fig. 1), nous observons un espace touristique en cohérence avec notre projet, pour deux raisons au moins.

D'une part, cette côte a été anciennement mise en tourisme avec Dieppe et les bains de la Duchesse de Berry dès les années 1824. En effet, les aristocrates français fréquentaient ce port 
d'embarquement pour l'Angleterre sur la route de Paris à Londres. Dieppe apparaissait alors comme un lieu d'importance dans le réseau de circulation français du début du XIX siècle et il a constitué, semble t-il, un foyer initiateur de développement touristique à une époque où bain froid et découverte du paysage correspondaient aux pratiques dominantes (Équipe MIT, 2005). En effet les paysages encadrant ce port rappellent ceux situés sur l'autre rive de la Manche. Le site d'Yport n'est-il pas identique à celui de Brighton? Et les aristocrates français exilés en Angleterre pendant la Révolution française n'ont-ils pas fréquenté de tels paysages?

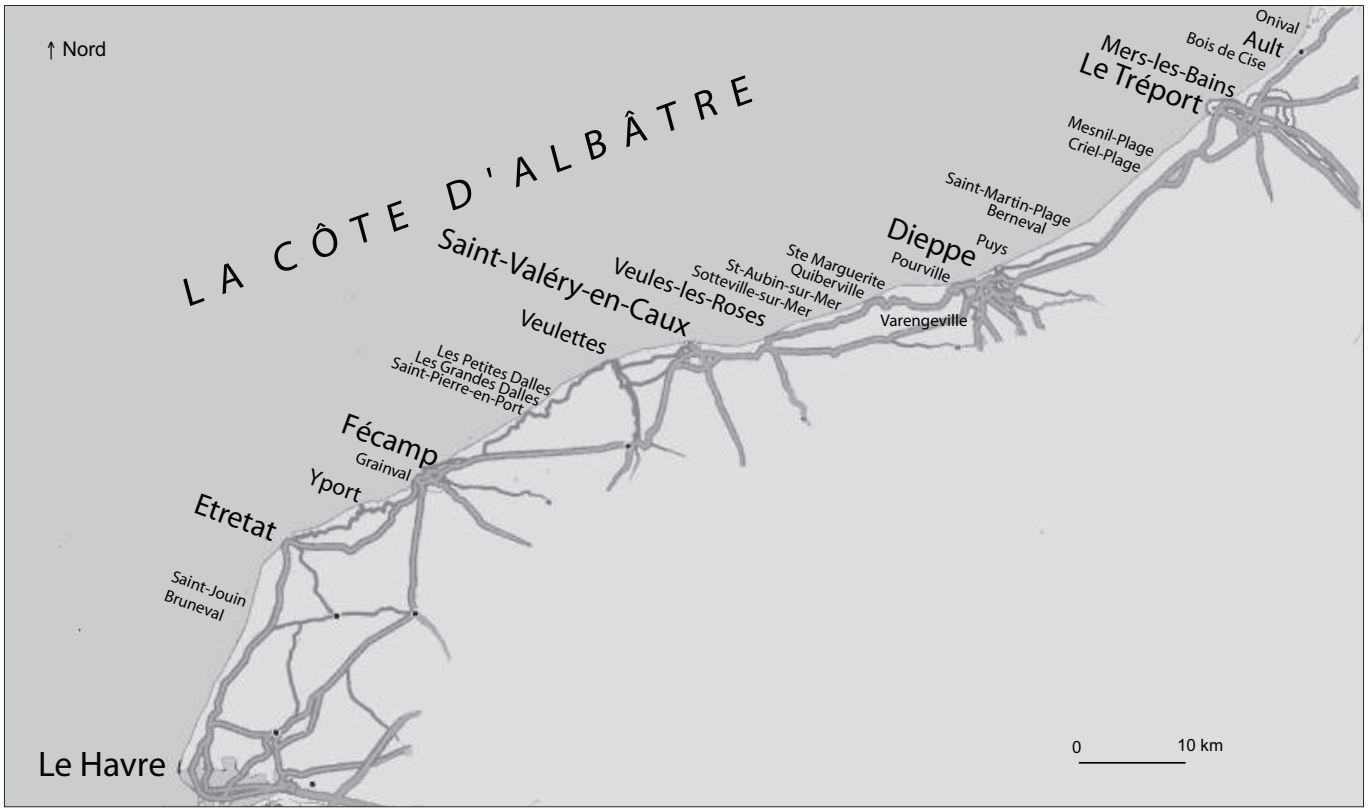

Figure 1 : La Côte d'Albâtre, d'Étretat à Ault The Côte d'Albâtre, from Étretat to Ault

D'autre part, cet espace n'est plus en vogue aujourd'hui, comme sorti de l'espace touristique national ou international, la côte touristique normande connue étant située plus au sud autour de Honfleur, Trouville-Deauville et jusqu'à Cabourg. Aujourd'hui la Côte d'Albâtre ne mobilise pas des flux touristiques massifs mais elle n'est pas vide de touristes pour autant. Alors ne seraitce pas là un espace constitué de " communautés vacancières "? La vie touristique semble, en effet, exister sans qu'elle corresponde pleinement aux images classiques que nous avons des lieux touristiques : front de mer bâti d'immeubles, large et vaste promenade de bord de mer, cohue de personnes et de voitures, démultiplication des services et des équipements...

\section{Et la Côte d'Albâtre fut}

Comprendre la situation touristique contemporaine de cette portion du littoral normand induit de nous tourner vers son histoire et de faire une lecture de sa mise en tourisme comme de son développement. Le premier temps de notre analyse portera sur la période 1842-1939. La première date se place près de quinze ans après le dernier voyage de la Duchesse de Berry à Dieppe et pendant le règne de Louis-Philippe, lequel a élu aussi cette côte en s'installant l'été à Eu et en pratiquant les bains au Tréport (Clary, 1977). On peut alors identifier les effets de ces présences sur le développement touristique. La seconde se situe juste avant le basculement touristique de cette côte comme nous l'évoquerons ci-après. 
Pour mener à bien notre travail, six guides ont été retenus : le Guide pittoresque, portatif et complet du voyageur en France (Saint-Fargeau, 1842), le Guide pratique aux Eaux minérales et aux bains de Mer (James, 1867), le Département de la Seine-Inférieure (Joanne, 1875), le guide des Plages du Nord et de la Normandie : de Dunkerque au Mont Saint-Michel (Bardet, 1892), le guide Diamant de Normandie de 1926 et le Guide Bleu Nord de la France, Flandre-Artois, Picardie de 1939. Nous y avons collecté une double information : la construction d'infrastructures touristiques et le discours sur le lieu.

\section{LA CONSTRUCTION D'UN ESPACE TOURISTIQUE}

Le Guide pittoresque, portatif et complet du voyageur en France (Saint-Fargeau, 1842) évoque seulement Saint-Valéry-en-Caux, Fécamp et Dieppe. Sont mentionnés des hôtels pour les deux premiers et Dieppe apparaît comme un lieu touristique où les bains sont parmi les « plus beaux en ce genre qu'il y ait en France » et un bel hôtel dispose d'appartements particuliers pour accueillir «plusieurs classes d'étrangers », signe que les séjours de la Duchesse de Berry ont eu leurs effets. Constantin James (1867) complète le tableau : Le Tréport, Mers-les-bains, Yport, Étretat font leur apparition. Une première hiérarchie s'instaure. Dieppe reste « une de nos plus célèbres plages balnéaires [...] » et Étretat surgit en quelques années pour devenir l'égal de Dieppe : « Dieppe et Étretat ont aujourd'hui une renommée à peu près égale. »

«C'est le peintre Isabey qui "découvrit" Étretat et qui la fit connaître à Paris par ses marines. Alphonse Karr la mit à la mode dans le monde littéraire et artistique et, sous la Monarchie de Juillet, c'est déjà un embryon de station avec quelques villas et pensions, gardant son cachet de petit port de pêche.

Sous le Second Empire elle grandit : un casino est construit et des routes la relient de Fécamp au Havre. Des visiteurs célèbres continuent à la fréquenter : peintres comme Delacroix, Corot [...], musiciens comme Offenbach, écrivains comme Maupassant, [...]. En 1871, elle double sa population et triple en surface. La digue est construite, le centre de la ville s'organise [...]» (Burnet, 1963).

Saint-Valéry-en-Caux apparaît comme dynamique dans les propos de C. James : ses « quelques hôtels à l'usage des baigneurs ». Les autres sont des lieux touristiques en constitution : Le Tréport est une ville où «à part des hôtels de bonnes apparences sont des maisons de pêcheurs » et le casino est « aussi silencieux le jour que la nuit » alors que « le casino de Fécamp est le plus grandiose que l'on puisse imaginer [mais] jamais lieu ne fut plus mal choisi pour une aussi splendide installation ». En effet, distant de la ville, la plage avec ses galets blessants et l'absence de distraction amènent l'auteur à conclure à l'insuccès en dépit de réels attraits balnéaires et à définir la ville comme « un port de cabotage » avant tout; Mers est « destiné, je crois, à devenir un bain important »; et le commentaire sur Yport reste ambigü : " grâce à ses bains de mer, Yport est un petit village où respire l'aisance et où quelques-uns de nos baigneurs ne craignent pas, [...] d'aller s'égarer».

Une décennie plus tard, A. Joanne (1875), dans son Département de la Seine-Inférieure, propose un classement précis des lieux et attribue le statut de «station de bains de mer » à neuf lieux : Mers, Le Tréport, Dieppe, Veules-les-Roses, Saint-Valéry-en-Caux, Veulettes, Fécamp, Yport et Étretat. Alors que le tourisme entre dans une phase d'industrialisation (Équipe MIT, 2005), la Côte d'Albâtre disposerait de neuf stations de taille et de renommée inégale, Veules et Veulettes surgissant promptement en quelques années. Cela s'expliquerait par l'apparition d'un casino, d'un établissement de bains et de quelques hôtels, comme le confirme le guide régional sur les Plages du Nord et de la Normandie (Bardet, 1892).

Dans cette publication, la plupart des lieux de notre échantillon est abordée et se différencie en deux types. D'une part, quatre stations dominent le paysage touristique de la Côte d'Albâtre : 
Le Tréport, Dieppe, Saint-Valéry-en-Caux et Étretat et tous les autres lieux sont définis comme leurs annexes. Un territoire en réseau émerge où Fécamp apparaît isolé, ne fédérant aucun lieu touristique aux alentours comme l'évoque le guide :

« La plage de Fécamp est triste, chargée de galets et la mer y est dure, aussi la réglementation des bains est-elle sévère. Deux établissements de bains et un petit casino reçoivent, au moment de la saison, un grand nombre de baigneurs; mais ceux-ci ont tendance à préférer les charmantes stations des environs, où l'on se trouve plus à la campagne que dans cette ville manufacturière » (Bardet, 1892).

Ainsi, Les Petites-Dalles, les Grandes-Dalles et Saint-Pierre-en-Port distants d'une dizaine de kilomètres de Fécamp, comme Saint-Valéry-en-Caux l'est de son annexe Quiberville, sont-ils présentés un ensemble homogène et autonome.

Le point d'apogée du développement touristique semble être la période suivante. Le guide Diamant de Normandie de 1926 montre une forte croissance des hôtels : de 62 en 1892 à 99 en 1926; 112 si on ajoute la partie intégrée à notre espace mais seulement présente dans le Guide Bleu du Nord de la France de 1939. Fécamp et Saint-Valéry-en-Caux marquent le pas (quatre établissements supplémentaires chacun) tandis que Dieppe, Le Tréport et Étretat doublent voire triplent leurs hébergements dont quatre hôtels grand luxe pour la première, un pour chacune des deux autres. Des lieux croissent : Ault (fig. 2), Mers, Veules ou Yport; d'autres enfin surgissent réellement sur la scène touristique : du Bois de Cise au sud d'Ault, Puys au nord de Dieppe (fig. 3) comme Pourville «petite station élégante » au sud de Dieppe avec huit hôtels construits dont un de « premier ordre ». Ce lieu semble avoir été très prisé au tournant du siècle tant par les artistes qui l'ont beaucoup peint comme Monet, en séjour avec sa famille, que par les musiciens tels que Debussy en 1904. Mesnil-Plage, Berneval, Sainte-Marguerite, Quiberville, Saint-Pierre-en-Port et Saint-Jouin-Bruneval avec trois ou quatre hôtels chacun tiennent une place secondaire mais progressent... Varengeville, Sassetot-Mauconduit et Les Grandes Dalles font leur apparition. Parmi ces petits lieux touristiques, d'autres marquent le pas comme Les Petites Dalles.

\section{LE DISCOURS SUR LE LIEU}

Cette première caractérisation permet de qualifier touristiquement un lieu et de le placer dans une hiérarchie. Toutefois, cette approche peut être complétée en observant les qualités de cette région touristique. Tout d'abord, il semble difficile d'y lancer de nouveaux lieux touristiques. G. Bardet (1892) rend compte de ces difficultés en évoquant Puys situé aux portes de Dieppe : « une tentative a été faite pour y établir un grand hôtel avec casino, mais le succès n'a pas jusqu'ici répondu à l'espérance des entrepreneurs et Puys reste une plage élégante mais calme ». Cela ne bloque pas pour autant les projets : " entre 1880 et 1913, 628 ha sont tenus par des sociétés; dans le Pays de Caux, un groupe anglais achète 57 ha en 1908 pour constituer Le Tréport-Terrasse, et un homme d'affaires parisien essaie de promouvoir 52 ha de friches dans les valleuses de Berneval-Saint-Martin; ailleurs (Étretat, Criel, Le Tréport, Ault-Woina-Rue), 27 ha sont mis à la disposition des bâtisseurs » et les Parisiens représentent à cette époque $65 \%$ des propriétaires et les habitants de la Basse-Seine $15 \%$ (Clary, 1977). Dans la période suivante (1915-1936), les projets se reconfigurent doublement par les acteurs qui les portent et par lieux investis : " Des hommes d'affaires locaux ont pris en mains le tourisme cauchois : ils [détiennent] $65 \%$ des 255 ha disponibles. La société anglaise a renoncé au Tréport-Terrasse, mais une partie de ses capitaux s'est reportée sur les Dalles, que tient cependant pour l'essentiel un groupe rouennais et Saint-Pierre-en-Port» (ibid.).

À cette première spécificité s'ajoute un discours particulier pour caractériser les lieux touristiques de la Côte. À l'exception de Dieppe reconnu très tôt comme un lieu d'envergure nationale, les autres lieux touristiques ne semblent pas appartenir à cette catégorie. Tout un vocabulaire en rend compte et Étretat l'incarne très tôt : 


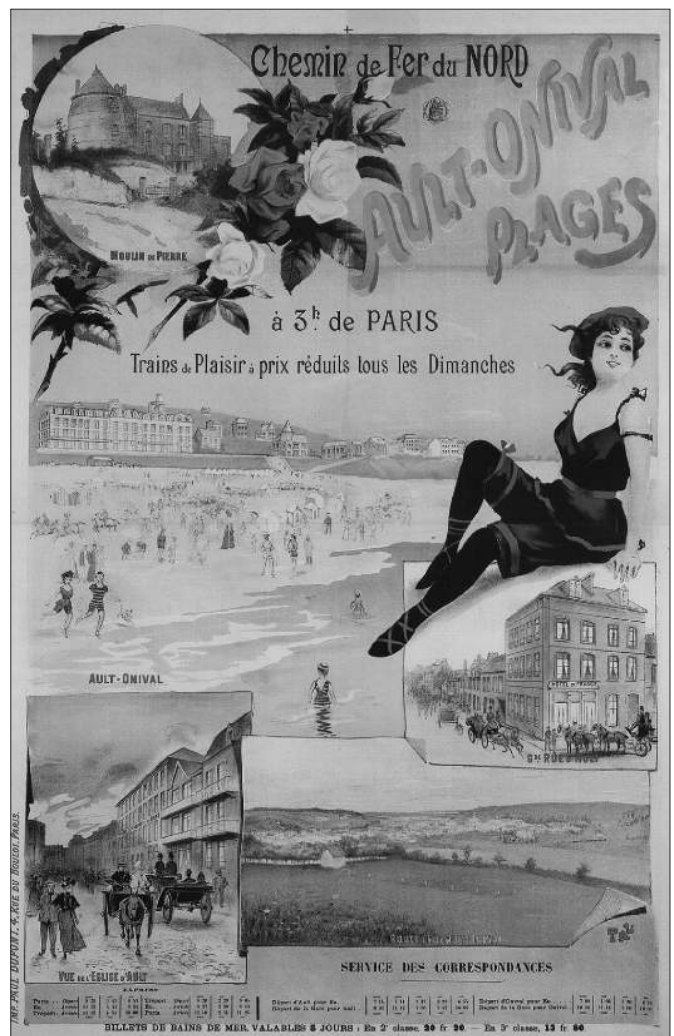

Figure 2 : Promouvoir en 1900 un lieu touristique aujourd'hui méconnu (Source : Château-musée de Dieppe, 2006, p. 53).

\section{unknown now}

Promoting a tourist place in 1900, resort

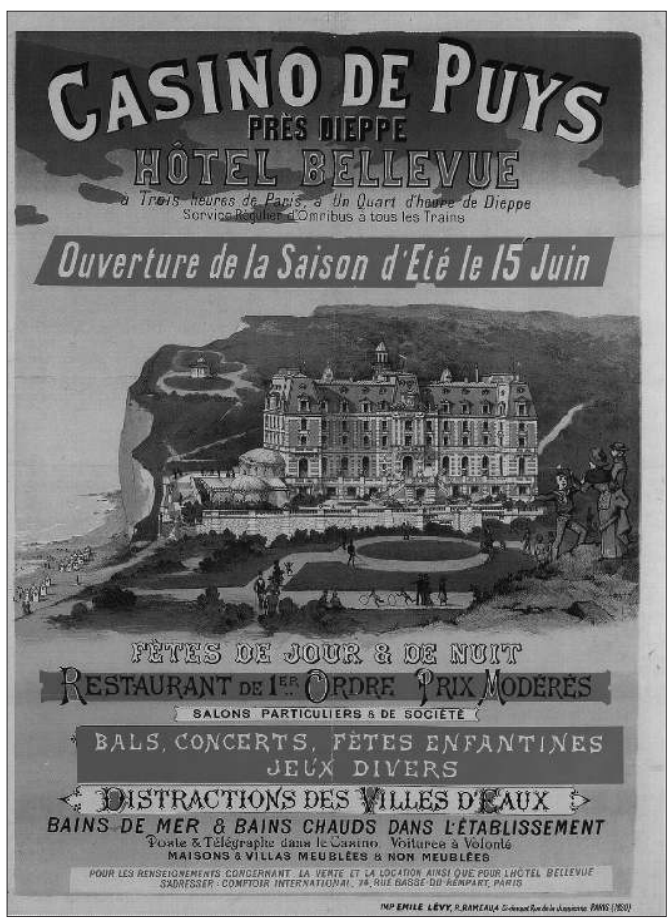

Figure 3 : L'inauguration d'un nouveau lieu touristique, Puys près de Dieppe (Source : Château-musée de Dieppe, 2006, p. 53)

Puys near Dieppe

The inanguration of a new tourist place,

«Étretat est le bain de mer des hommes de lettre et des artistes. [...] Ne cherchez ni casino somptueux, ni toilettes élégantes; il y a plutôt des réunions intimes que des réceptions d'apparat. [...] Les hôtels eux-mêmes ont un aspect quelque peu primitif : c'est que tout baigneur a sa maison au milieu des champs et de la verdure. »(James, 1867)

Ce discours montre l'existence de lieux touristiques fondés sur d'autres ressorts. Analyse confirmée par G. Bardet (1892) qui décrit les lieux selon deux corpus de vocabulaire : si Le Tréport est "mondaine », «la tranquillité [d'Ault] et l'absence totale de luxe en font un séjour très agréable pour les familles qui aiment le calme et le bien-être moral », tout comme Berneval et Saint-Valéryen-Caux où la «vie est simple et facile », tout comme les Petites-Dalles et les Grandes-Dalles : «stations [...] calmes et tranquilles et [...] surtout habitées, pendant la saison des bains, par des familles » (1892, p. 154). Et l'auteur de conclure son texte par cette déclaration à propos d'Étretat qui incarne, encore à l'époque, un certain type de stations :

« [II] faut reconnaître que, tout en étant mondaine, la vie d'Étretat diffère sensiblement de celle qu'on mène à Dieppe et à Trouville; le public n'est plus le même, c'est celui des premières de Paris, c'est-à-dire des artistes, des hommes de lettres, des financiers, des savants, des hommes politiques, tandis que, dans les grandes stations que nous venons 
de citer, on trouve surtout les grandes mondaines, les amateurs de courses et toutes les personnes qui mènent la grande vie et transportent volontiers le Bois de Boulogne et le boulevard partout où ils se rendent, que ce soit au bord de la mer ou dans les villes d'eaux à la mode. » (Bardet, 1892)

Dans cet ouvrage, le discours sur les prix reprend autrement cette idée. D’un côté se trouvent les stations chères : Fécamp est la plus chère pour les bains (8,40 francs la douzaine) devant Étretat ( 7 francs) et Dieppe ( 5 francs). L'abonnement mensuel au Casino est un autre signe de distinction : 60 francs pour Dieppe, 45 pour Étretat, 38 pour Le Tréport, et 25 francs à SaintValéry-en-Caux. Pour les autres, la vie apparaît comme «bon marché », « facile et peu coûteuse » à Berneval, ou encore « moins élevés [à Criel] qu'au Tréport ». Deux mondes touristiques cohabitent sur la Côte d'Albâtre.

Enfin, le guide Diamant de 1926 pérennise cette idée en qualifiant près de la moitié de notre échantillon de stations «petites », «paisibles », « charmantes » ou « modestes »; d'autres n'ayant aucune qualité touristique en dépit de la présence d'hôtels comme Varengeville : « renommée pour ses superbes allées de grandes arbres » ou Sainte-Marguerite et ses trois hôtels : «village à l'ouest duquel la Sâane se jette dans la mer », commentaire qui laisse songeur.

Pourtant, la Côte d'Albâtre était touristique dès le tournant du XX siècle et l'évolution de l'entredeux-guerres ancrait encore davantage cet espace dans cette réalité, même si de réelles différences existaient selon les destinations littorales. Aujourd'hui, à y regarder, les choses semblent avoir changé.

\section{Un double changement de Monde}

Et tout particulièrement depuis le Seconde guerre mondiale, période où deux événements majeurs ont contribué à transformer touristiquement la Côte d'Albâtre : les effet de la guerre et tout particulièrement des destructions d'une part et la révolution du « chaud » dans les pratiques touristiques d'autre part.

\section{DESTRUCTIONS}

Tout d'abord, le mur de l'Atlantique construit par les Allemands pour prévenir toute invasion possible joue, sans qu'on l'imagine toujours, un rôle dans l'évolution des lieux touristiques qui nous occupent. En effet, afin qu'aucun point précis de la côte ne soit observable depuis la mer, les Allemands détruisirent des hôtels de front de mer, éliminant non seulement tout point de repérage mais également les emblèmes touristiques de ces lieux. Ainsi en fut-il de l'Hôtel de bains aux Petites Dalles. D'autre part, les destructions liées à la guerre ont participé de cette œuvre de transformation radicale qui a concerné l'ensemble de la Côte d'Albâtre : «En effet, de nombreuses villas de front de mer sont détruites car elles gênent la défense du littoral et elles essuient les tirs de barrage des débarquements. Des coupes sévères ont lieu : [...], Berneval et Dieppe [sont détruites] à $40 \%$ \% (Clary, 1977).

Tout cela aurait pu être temporaire et les stations de bord de mer en partie détruite auraient pu renouer avec le passé et retrouver un aspect touristique avec une inscription architecturale nouvelle. Pourtant ces destructions semblent avoir sonné le glas touristique de ces stations car, à partir de 1945, l'urgence était ailleurs et l'ambiance générale guère concernée par le tourisme : « La région met longtemps à panser ses blessures et la bourgeoisie urbaine à songer à ses aires de loisirs : il lui faut d'abord rebâtir des villes et se donner des activités » (ibid.).

Cela expliquerait l'évolution des différents fronts de mer des stations de la Côte d'Albâtre. Tout d'abord, certains ne sont pas reconstruits selon des logiques touristiques. Au Tréport, la plage est bordée d'une promenade en surplomb dominant la mer d'un côté et de l'autre côté parkings et routes auxquels succède un immeuble massif par sa longueur plus que par sa hauteur, réservé à la 
résidence principale (photo 1 et 2). Il fallait loger rapidement les sinistrés et on ne recrée pas les conditions de la déambulation et de la rencontre, si précieuses à la vie d'une station touristique (Équipe MIT, 2002).

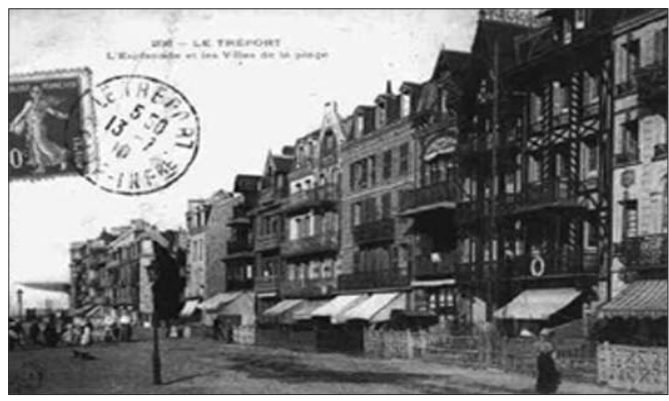

Photo 1 : L'esplanade des villas au Tréport au début du $\mathrm{XX}^{\mathrm{e}}$ siècle (Source : http//www.notrefamille.com)

The "esplanade des villas": Le Tréport at the beginning of the $20^{\text {th }}$ century

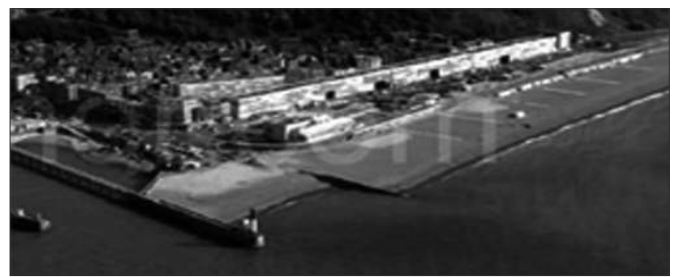

Photo 2 : Le front de mer au Tréport aujourd'hui (Source : Paris-Normandie)

\section{Le Tréport sea front nowadays}

Ailleurs il n'y a pas eu de reconstruction ou plutôt seules quelques opérations ont été réalisées au fil du temps, conférant au front de mer une silhouette hachée, sans commune mesure avec la période précédente. Pourville (commune d'Hautout sur mer) en est un exemple car il montre combien le bord de mer reste inoccupé de construction même si la trame viaire de la station apparaît très précisément. En revanche, la photographie proposée ne montre pas la densité de villas longeant le versant sud-est de la valleuse, témoin d'une villégiature passée et présente encore forte (photo 3). Aux Petites Dalles, seul l'Hôtel des Bains disparu n'a pas été reconstruit, la préférence allant à la réalisation d'un parking (photo 3 ).

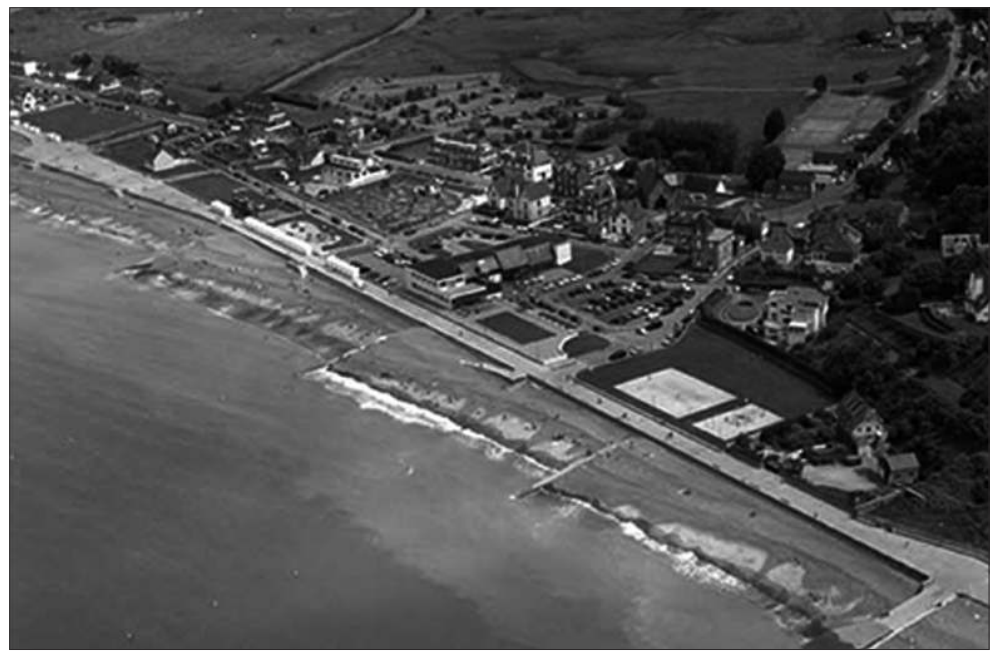

Photo 3 : Le front de mer de la station de Pourville Source : Paris-Normandie sea front Pourville resort

D'autres lieux n'étaient sans doute pas encore suffisamment construits avant guerre pour qu'ils survivent à ces mutations. Malgré l'absence des dommages de guerre, ils ne suscitèrent plus le même intérêt comme Saint-Pierre en Port (photo 6). Là, toujours pas de front de mer et un lieu peu construit. 

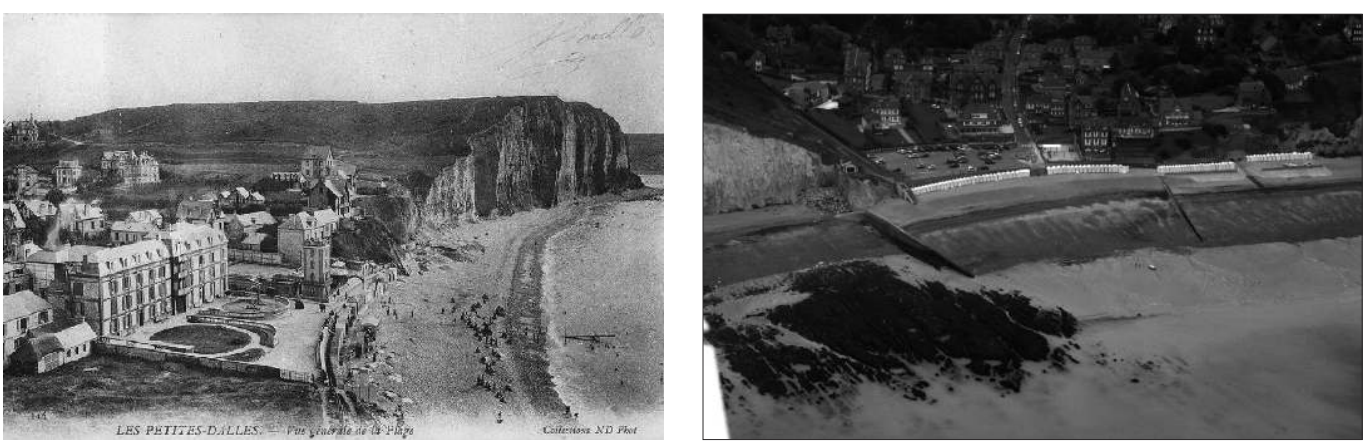

Photo 4 et 5 : L'évolution du front de mer aux Petites Dalles : quand le parking succède au Grand Hôtel des Bains (Source : [http://www.les-petites-dalles.org] et Paris-Normandie

des Bains

The sea front evolution at Les Petites Dalles: when a parking takes the place of the Grand Hôtel

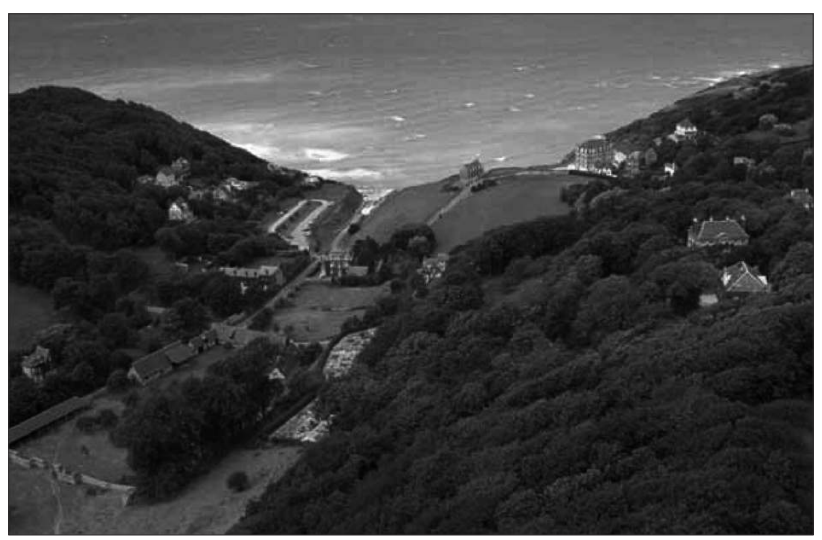

Photo 6 : Un front de mer qui n'a jamais existé à Saint-Pierre-en-port

A sea-front which has never

existed, St Pierre en Port

\section{LA MUTATION DES PRATIQUES TOURISTIQUES}

De plus, cette côte a subi de plein fouet le basculement des valeurs touristiques dans l'Entredeux-guerres. La révolution "américaine » qui se déroule dans les années 1920 à Juan-les-Pins (Équipe MIT, 2005) porte le bronzé, le soleil et le chaud au firmament des moteurs de la pratique touristique. Dorénavant, les lieux qui cumulent ces attraits seront plébiscités ou auront toutes les chances de l'être, reléguant touristiquement les lieux fondés sur le pâle et le froid.

«Si Juan-les-Pins et le Cap d'Antibes forment un moment de lieu dans les années 1920, ce n’est pas pour l'invention de la fréquentation de la plage l'été. Dès le début des années 1920 en effet, presque toutes les stations de la côte atlantique [...] sont fréquentées essentiellement l'été. Seules les plus grosses, comme Biarritz, Arcachon, Royan ou les Sables-d'Olonne, sont fréquentées à l'année. Mais il s'agit là d'un glissement de la saison des bains, qui se prennent encore à l'automne à la fin du XIX siècle, allant de pair avec le développement de stations familiales et l'affaiblissement du discours médical sur les eaux froides. N'oublions pas que le bain de mer est l'activité fondatrice du tourisme sur la façade atlantique. En Méditerranée, en revanche, c’est la douceur du climat qui est à l'origine du développement touristique. 
Aussi le début d'une fréquentation estivale en Méditerranée correspond bien à un passage important. Il ne s'agit pas d'une simple transition, mais d'un véritable basculement du frais vers le chaud, de l'ombre vers le soleil, du pâle vers le halé. Un basculement qui allait définir (devenir?) la référence dans le monde entier. Il semble bien que les Américains, d'abord aux États-Unis [...] puis en France, aient joué un rôle décisif dans cette émergence. » (Équipe MIT, 2005)

Les flux touristiques changent d'orientation et si l'Atlantique a pu tirer son épingle du jeu comme la citation l'évoque, cela fut plus difficile pour des lieux situés aux horizons septentrionaux de la France bordant une mer froide et reconnue comme telle : la Manche qui, à quelques exceptions près (Le Touquet, Deauville-Trouville, Saint-Malo-Dinard), ne regroupe pas une côte touristique densément aménagée et fréquentée. Ainsi assiste t-on à un repositionnement touristique de la Côte d'Albâtre : "La notion de plage est associée spontanément de nos jours à celle de sable. La vogue des stations cauchoises date d'une époque où la vie mondaine comptait plus que la pratique des sports dans la nature et où la proximité de Paris était fondamentale. Aujourd'hui, la rapidité des transports et la villégiature de masse aiguillent vers d'autres lieux les estivants » (Burnet, 1963).

Sans formuler l'analyse de la même manière, nous voyons combien le tourisme change aux lendemains de la Seconde Guerre mondiale et privilégie des espaces immédiatement utilisables, alors que le littoral cauchois ne sera que progressivement reconstruit. Ainsi le quartier des Bains de Saint-Valéry-en-Caux, détruit en 1940, ne sera-t-il achevé qu’en 1964. Trop tard.

\section{LA CÔTE D’ALBÂTRE : UN ANGLE MORT DANS L'ESPACE TOURISTIQUE ?}

Aussi la capacité d'accueil globale est-elle très réduite aujourd'hui. Sur l'ensemble observé se concentrent près de 60000 lits touristiques auxquels il faudrait ajouter les lits en chambre d'hôtes. La médiane est à 1800 lits et quelques lieux forts dominent aujourd'hui comme hier : Dieppe et Fécamp, Le Tréport et Mers-les-Bains (13000 lits), Criel-sur-Mer et Ault (plus de 5000 lits).

Les hébergements marchands sont très peu présents. 13500 lits repartis sur 32 communes dont seulement 24 proposent hôtels et/ou campings. Cette situation indique donc des contrastes très grands selon les lieux même si tous ont été marqués par une forte dynamique de recul depuis l'après-guerre. Ainsi, le nombre d'hôtels montre un recul significatif : de 112 en 1926 à 65 en 1961 et 59 en 2005. Les ville-stations et grandes stations sont fortement touchées : Dieppe passe de 36 hôtels en 1926 à 12 aujourd'hui, Le Tréport de 15 à cinq et Saint-Valéry-en-Caux de dix à cinq également. À une autre échelle, l'ensemble Ault-Onival-Bois de Cise de huit à un hôtel comme Pourville, mais Étretat se maintient à 11-12 hôtels montrant ainsi la pérennité de son attrait paysager. Dans le même temps, les campings se sont développés. On compte près de 11400 lits alors qu'il n'en existait pas en 1961. En revanche, la Côte d'Albâtre est l'espace des résidences secondaires. Avec près de 50000 lits, elles représentent près $75 \%$ de l'hébergement touristique.

D'autre part, l'évolution des guides depuis l'après-guerre montre une sélection de plus en plus forte entre les lieux. L'espace touristique se recompose, délaissant les uns, concrétisant les autres. Le Guide bleu de Normandie (1961) apparaît comme le plus complet historiquement, dans la mesure où il évoque tous les lieux pris dans une dynamique touristique de séjour : même Grainval près d'Yport est évoqué comme «petite station balnéaire ».

Le Guide du Routard sur la Normandie (1998-1999) propose une lecture différente et intéressante de la Côte d'Albâtre car de nombreux lieux, évoqués ou détaillés préalablement disparaissent ${ }^{1}$. D'autres deviennent plus centraux dans notre échantillon ${ }^{2}$ et deux exemples sont

1. Ault-Onival alors que Bois de Cise est évoqué ; Criel et Berneval; Puys et Pourville; Quiberville et Sotteville; SaintPierre-en-Port, Grandes Dalles et Sassetot-Mauconduit; Vaucottes et Saint-Jouin-Bruneval.

2. Nous ne parlerons pas ici des lieux nouveaux qui apparaissent, car cela reviendrait à traiter la dynamique de l'espace touristique à travers les guides, ce qui n'est pas notre propos ici. 
intéressants à noter. D'une part Étretat est devenue «presque une légende, tant l'arche de craie plongeant dans la mer fait partie des images les plus ancrée dans nos mémoires ». C'est sans doute le lieu qui draine depuis la France et l'Europe entière des touristes, de passage. D'autre part, Varengeville-sur-Mer ignorée jusqu'au Guide Diamant de 1926 où l'on précisait qu'elle était « renommée pour ses superbes allées de grands arbres » et qu'elle disposait d'un hôtel est devenu progressivement un lieu d'intérêt dès 1961 :

« dispersé sur un plateau boisé, n’a pour ainsi dire pas de centre communal. Plusieurs hameaux sont disséminés le long de chemins ombragés de beaux arbres, qui forment un véritable dédale. C'est un lieu de villégiature champêtre plutôt que balnéaire, fréquentée par une colonie d'artistes et d'écrivains qu'a séduits ce site agreste » (Guide Bleu, 1961).

«Atmosphère champêtre et bourgeoise à la fois, grâce aux superbes demeures du début de siècle bien camouflées derrière des écrans d'arbres. C'est là tout le charme. Varengeville a toujours été célébré par les artistes. Braque et Queneau adoraient l'endroit. Miro captivé par les cieux étoilés y séjourna de 1937 à 1940 et s’inspira de ce coin pour réaliser son cycle Constellations et ses œuvres Varengeville 1 et Varengeville 2 » (Guide du Routard, 1999).

Au total, par ce premier travail sur le temps long, nous saisissons les avancées comme les replis touristiques qui ont affectés les lieux constitutifs de la Côte d'Albâtre. On identifie mieux aussi les dynamiques liées au marché touristique (hôtel et camping) des dynamiques non-marchandes avec les résidences secondaires, et les lieux évoqués on non par les guides. Tout cela constitue autant de critères favorables à l'identification des communautés vacancières de ce littoral.

\section{Identifier les communautés vacancières de la Côte d'Albâtre}

En effet, par la reconstitution de l'itinéraire touristique de la Côte d'Albâtre, il nous est possible d'entreprendre l'identification des communautés vacancières. Pour cela, sélectionnons-les à partir des critères touristiques.

\section{CONSTITUTION D’Un ÉCHANTILLON}

Tout d'abord, on recherche des lieux ayant une activité touristique. Elle peut être identifiée par le nombre de lits touristiques et le taux de fonction touristique. La faiblesse de l'un va généralement avec la faiblesse de l'autre ici et permet de supprimer dans un premier temps sept lieux ${ }^{3}$. Doivent être aussi retirés les lieux jamais évoqués dans les guides dépouillés : Belleville-sur-mer serait le seul dans ce cas.

Ensuite doivent être écartés de l'analyse les lieux touristiques d’importance par la présence d'hébergements marchands, signifiant une ouverture par le renouvellement de la population touristique que cela induit. On doit donc supprimer les trois villes à fonction touristique que sont Dieppe, Fécamp et Saint-Valéry-en-Caux, caractérisées par une forte capacité d'accueil doublée d'une importante population résidente. De même les stations touristiques de la Côte d'Albâtre sont à écarter du raisonnement. Trois critères sont retenus ici : un taux de fonction touristique supérieur ou égal à 1 , une part des résidences secondaires dans le total des hébergements relativement réduite au regard de la moyenne de la Côte, et un nombre élevé d'habitants permanents. Ces

3. Belleville-sur-mer, Bracquemont, Criquebeuf en Caux, Életot, Penly et Saint-Jouin-Bruneval. Le taux de fonction touristique n'atteint jamais 0,5 et le nombre de lits n'excède pas 300 . 
deux derniers critères ne favoriseraient pas l'entre-soi recherché par les résidents de communautés vacancières. Sept lieux sont alors écartés de l'échantillon (tableau 1).

\begin{tabular}{|c|c|c|c|c|}
\hline $\begin{array}{l}\text { Nom de la } \\
\text { commune }\end{array}$ & Nombre d'habitants & $\begin{array}{l}\text { Nombre de lits } \\
\text { touristiques }\end{array}$ & $\begin{array}{l}\text { Taux de } \\
\text { fonction } \\
\text { touristique }\end{array}$ & $\begin{array}{c}\text { \% des résid. } \\
\text { secondaires } \\
\text { dans le total des } \\
\text { hébergements }\end{array}$ \\
\hline Le Tréport & 5900 & 6600 & 1,1 & 74 \\
\hline $\begin{array}{l}\text { Mer-les- } \\
\text { Bains }\end{array}$ & 3400 & 6500 & 1,9 & 86 \\
\hline Criel-sur-Mer & 2700 & 5100 & 1,5 & 85 \\
\hline $\begin{array}{l}\text { Hautot-sur- } \\
\text { mer }\end{array}$ & 2100 & 2100 & 1 & 49 \\
\hline Ault & 2000 & 5500 & 2,7 & 88 \\
\hline Étretat & 1600 & 3100 & 2 & 77 \\
\hline Yport & 1000 & 2000 & 2 & 58 \\
\hline
\end{tabular}

Tableau 1 : Les stations touristiques de la Côte d'Albâtre (Source : élaboration personnelle à partir du RGP 1999)

The Côte d'Albâtre tourist resorts

\section{UNE PREMIÈRE IDENTIFICATION}

Alors, les autres lieux appartiendraient aux communautés vacancières. En quoi se distinguentelles des autres lieux touristiques préalablement cités? Le taux de fonction touristique double presque passant de 1,3 à 2,4 dans la mesure où la faible masse des hébergements (17300 lits) s'impose face à une population résidente très réduite (8 575 personnes) avec seulement trois communes atteignant 1000 habitants. Ici tourisme et touristes sont en nombre face à des habitants permanents peu nombreux. Les communautés vacancières seraient donc des lieux touristiques.

De plus le nombre de lits banalisés tombe à près de 3800. En 2005, selon les statistiques du Ministère du tourisme, Varengeville (figure 1) n'a pas de camping, quatre autres communes n'ont pas d'hôtels (Berneval-le-Grand, Saint-Martin-en-campagne, Saint-Pierre-en-Port et Veules-lesRoses) et quatre ne disposent ni de l'un ni de l'autre (Bénouville, Sainte-Marguerite-sur-mer, Quiberville-sur-mer et Vattetot-sur-mer) alors que tous les autres lieux touristiques du Pays de Caux cumulent les deux, sauf Dieppe (pas de camping) et Pourville (commune d'Hautot-sur-Mer qui ne possède pas d'hôtels). Cela traduit un recul généralisé des hébergements marchands et tout particulièrement de l'hôtellerie qui passa de 31 établissements en 1926 à 7 en 2005, totalisant 194 lits, c'est dire la petitesse de ces hôtels qui atteignent au mieux une qualification en deux étoiles. En revanche 18 campings ont été créés, totalisant 3600 lits en 2005, dont quelques-uns sont trois et quatre étoiles tels que ceux de Berneval et Saint-Aubin-sur-mer.

Aussi la caractéristique de ces lieux est la forte présence des résidences secondaires. Avec près de 13500 lits, elles représentent $80 \%$ de l’hébergement touristique contre $74 \%$ pour les stations et villes touristiques. Selon les communes, le poids varie de $39 \%$ à $100 \%$ de l'hébergement et dépasse régulièrement 1000 lits. Cette prégnance de la résidence secondaire dans des lieux peu habités rend compte de ce qui pourrait caractériser la communauté vacancière : le séjour de personnes dans leur maison particulière, ou prêtée plus que louée à des amis, connaissances, parentèles...

Ce poids de la résidence secondaire peut être amplifié si l'on a recours à d'autres critères. En effet, dans les statistiques fournies par le Ministère du tourisme, est proposé le nombre d'emplacements de campings loué à l'année. Il s'agit de personnes qui laissent leur caravane, ou installent 
un mobil-home, une habitation légère de loisirs. C'est une forme de résidence secondaire, moins coûteuse que l'achat d'une maison, mais qui relève du même processus : l'investissement dans un lieu où l'on souhaite revenir régulièrement. Une fidélisation à la destination.

Ce mode d'investissement distingue les villes et les stations des communautés vacancières avec $27 \%$ du total des emplacements pour les premières et $40 \%$ pour les secondes. Ici s'observent plusieurs dynamiques intéressantes. D’une part Dieppe, Fécamp et Le Tréport proposent l'ensemble de leurs emplacements à une clientèle de passage. En revanche, $65 \%$ du total est loué à l'année à Pourville, Ault et Yport. Il s'agit vraisemblablement de stations où le degré de fidélisation de la clientèle est très fort. Une station d'habitués qui mériterait aussi qu'on s'y intéresse pour comprendre les modalités de fonctionnement de tels lieux et voir si, à leur manière, ils ne s'apparentent pas à une communauté vacancière. Ce travail reste à faire.

Pour notre échantillon, seule Veules se structure comme une station avec seulement $7 \%$ des emplacements loués à l'année. Cela nous inciterait à revoir sa place au sein des communautés vacancières. Sinon les niveaux les plus bas sont à $20 \%$; Saint-Martin-aux-Buneaux atteint $40 \%$, Saint-Pierre-en-Port comme Saint-Aubin-sur-Mer dépassent les $60 \%$ et Sotteville-sur-mer louent annuellement tous ses emplacements.

Cette lecture double de la résidence secondaire (maison et camping) dans l'hébergement touristique de certains lieux de la Côte d'Albâtre montre un espace de très forte appropriation par des touristes, un véritable territoire touristique (Équipe MIT, 2000) où $90 \%$, en moyenne, des hébergements sont des propriétés privées.

\section{L'image et le fonctionnement d'un type de lieu}

Alors à quoi ressemblent de tels lieux? Car l'expression physique traduit une organisation, un fonctionnement et permet d'enrichir le propos. L'image proposée par la plupart de ces lieux confine davantage à une urbanisation de maisons individuelles, villas anciennes de la «villégiature » ou plus récentes liées à la reconstruction d'après-guerre. Une certaine homogénéité du bâti existe, expression du désintérêt pour le lieu ou de l'impossibilité d'y construire. Pour beaucoup, l'urbanisation suit la configuration de la valleuse dans laquelle le lieu se déploie et prend deux formes: soit un développement sur l'un des versants comme à Saint-Pierre-en-Port (photo 6), soit l'investissement du fond et des côtés comme aux Petites Dalles. Parfois, elle se développe sur l'ancien village à l'entrée de la valleuse comme Varengeville. Généralement les campings se sont installés sur le plateau.

Le front de mer est sans doute l'un des éléments les plus emblématiques des communautés vacancières car il ne correspond en rien à l'image que nous nous en faisons actuellement. Ici, une absence des lieux traditionnels de la sociabilité touristique est manifeste : point de Casino, une promenade assez réduite et limitée au débouché du vallon et localisée devant le parking. Ce dernier accentue cette piètre disposition du bord de mer pour la rencontre. De plus, il y a souvent une distinction architecturale entre le littoral, plus récent, et l'intérieur plus « villégiature balnéaire ». Et l'absence de pression immobilière forte n'a entraîné ni son évolution, ni sa destruction : pas de verticalisation du front de mer marquant la réussite du lieu comme cela été le cas dans d'autres lieux comme Nice, Le Touquet ou La Baule mais aussi Brighton ou Scarbourough.

\section{Le cas des Petites Dalles, un exemple pour saisir un type de lieu}

L'entretien que nous avons eu avec Pierre Wallon ${ }^{4}$ aux Petites Dalles en avril 2006, permet de mettre en perspective l'ensemble de notre analyse. Il constituera notre dernier élément d'analyse.

4. Pierre Wallon est le descendant d'Henri Wallon : auteur de l'amendement Wallon qui proposait que le Président soit élu à la majorité absolue des suffrages des membres du Sénat et de la Chambre des Députés réunis. Voté en Janvier 1875 à une voix de majorité, il confirme la nature républicaine de la IIIe République. Pierre Wallon nous a reçu dans sa villa « Les Chrysanthèmes ». Les propos mis entre guillemets sont les siens, consignés au cours de 
En effet, à l'écouter nous raconter l'histoire des Petites Dalles, on apprend déjà que le casino des Petites Dalles qui avait brûlé en 1904 ne fut pas reconstruit. Et lorsque les Allemands détruisirent le Grand Hôtel des Bains, le grand père de Pierre Wallon ne voulut pas qu’on le reconstruise pour « ne pas ouvrir la station aux mondanités ». Ce propos est intéressant car, très tôt (dès 1904), tout est fait pour que les mondanités ne soient pas maintenues dans le lieu et de ce point de vue, les destructions allemandes peuvent apparaître a posteriori comme une aubaine. La disparition de l'hôtel, comme celle du Casino, sonne le glas des lieux de la mondanité et de la venue d'éventuels extérieurs. La tranquillité est confirmée, voire renforcée.

Cet entre-soi, cette confidentialité est au cœur du fonctionnement de la communauté vacancière, une posture presque co-substantielle du lieu. Nous l'avons déjà évoquée à travers les discours des guides, de C. James (1867) ou même de G. Bardet (1892), où on fuyait les "mondanités ». Aussi les communautés vacancières sont issues, en partie, de stations touristiques accueillant des populations touristiques ne souhaitant pas fréquenter les lieux en vogue (Dieppe, Trouville) mais désirant une tranquillité. Même si le tourisme fonctionne sur la rencontre de l'Autre (Ceriani et al., 2005), les populations ont des désirs variés face à cette rencontre et elle peut s'exprimer par la recherche de lieux de taille réduite, de diversité sociale limitée, d'un entre-soi très poussé tant du point de vue social que quantitatif.

Dans le cas des Petites Dalles, quelques familles ont participé au développement touristique du lieu par l'édification de nombreuses maisons et la famille Wallon est de celles-là. L'entre-soi des communautés vacancières est alors plus poussé qu'on ne pouvait le croire car il peut, comme ici, relever d'un projet de quelques acteurs qui souhaitent construire leur propre lieu touristique, non loin de Paris pour se rendre sur place facilement; une bourgeoisie fortunée qui « avait une tradition de retenue » : «On n'allait pas se montrer et on aimait être entre soi. » Ainsi se forgeait une autre sociabilité fondée sur les rencontres et les alliances. Il était/est « très habituel » que les enfants des différentes familles présentes se marient entre eux. Et la sociabilité s'opère davantage sur la plage ou au tennis-club et à l'heure du café, distinguant même au sein des Petites Dalles deux groupes : ceux qui vont à la plage et ceux qui se rendent au tennis-club.

«Pour vivre heureux, vivons cachés » serait sans doute le slogan fonctionnaliste des communautés vacancières. Idée illustrée par les réactions des résidents secondaires lorsqu'un responsable du syndicat d'initiative avait décidé d'animer les Petites Dalles avec un feu d'artifice : il fut renvoyé rapidement. Aujourd'hui, l'existence d'un site internet proposant des photographies anciennes des Petites Dalles ${ }^{5}$ a valu à son créateur, Pierre Wallon en l'occurrence, des réactions peu amènes. Ces postures et réactions font écho à ce que les touristes attendent toujours du lieu où ils séjournent, qu'il soit hors des bruits du monde, hors du monde. Là on en identifie une facette originale.

\section{En guise de conclusion : communautés vacancières, touristiques ou recréatives?}

Notre travail a permis de mieux identifier et comprendre le fonctionnement d'un certain type de lieu touristique. Il s'agit de lieux particuliers dans la mesure où ils ont été investis voici longtemps par les touristes et le marché, puis déserté par ce dernier ou réduit à une portion congrue. La résidence secondaire, maisons ou campings, s'affirme plus qu'ailleurs pour devenir localement omniprésente. Cela induit la présence de touristes qui recherchent un entre-soi, ce qui est commun au tourisme. Mais ici il est poussé puisque le lieu sera réservé à quelques-uns, dont la présence est fondée souvent sur l'ancienneté de l'installation touristique, la volonté de ne pas faire connaître leur lieu à l'extérieur. À l'image de quelques destinations touristiques, il s'agit de " lieux réservés », mais pas systématiquement à une élite. L'histoire mondiale et touristique a favorisé l'émergence

l'interview. D'autres grandes familles bourgeoises sont présentes dans cette région comme les Servan-Schreber à Veulettes où fut récemment inhumé J.-J. Servan-Schreber.

5. [http://www.les-petites-dalles.org] 
de ce type de lieux sur la Côte d'Albâtre, mais gageons qu'il en existe ailleurs sur des littoraux plus en vogue touristiquement. Il y a là une réflexion à poursuivre, notamment en observant les stations dites «familiales », viviers peut-être de « communautés vacancières ».

Nous avons appelé ce type de lieu « les communautés vacancières ». À l'issue de notre réflexion, nous aimerions reprendre cette expression et la questionner pour mesurer sa pertinence face à deux autres appellations possibles : « communautés touristiques » et « communautés recréatives ». Comme évoqué en introduction, le mot «vacances » reste vague, exprimant des réalités variées sur lesquelles il est assez difficile de forger une terminologie sérieuse. Pourtant cela nous a aidé, pendant une période, à réfléchir sur ce type de lieu. Recréatif, selon notre travail, lie à la fois le tourisme et les loisirs (Équipe MIT, 2002, p. 103-126). Dans les lieux qui nous occupent, il y a des usages recréatifs de l'espace puisque, par exemple, la plage des Petites Dalles est pratiquée par les résidents secondaires, les touristes qui séjournent alentours ou les résidents permanents des villes et des villages voisins. Il s'agit d'espaces recréatifs mais cela ne peut s'appliquer à la communauté, qui n'est pas dans une démarche de loisirs lorsqu'elle est présente mais dans une démarche touristique. Aussi, l'appellation de « communautés touristiques » nous apparaît comme la plus probante car les personnes présentes sont des touristes. Ces touristes sont presque exclusivement des résidents secondaires, des touristes pas comme les autres (Duhamel, 2000).

Tout cela nous permet alors de proposer une première définition de la communauté touristique : c'est un lieu créé et dominé par le tourisme ${ }^{6}$, une création ex nihilo dont les caractéristiques rappellent grandement la station mais en diffèrent par l'omniprésence de la résidence secondaire, même si un hébergement marchand peut se maintenir à un niveau bas. Le développement et la pérennité du lieu reposent sur quelques acteurs, voire quelques familles et leurs réseaux. En cela, il ne s'agit pas d'un lieu ouvert sur la société locale ou globale. Le lieu se veut réservé à quelquesuns pour assurer leur tranquillité, leur « entre-soi ».

\section{Sources}

Château-Musée De Dieppe, 2006, Affiches touristiques de Dieppe et de sa région, 1880-1970, Ministère de la Culture et de la Communication/Ville de Dieppe, 79 p.

Guide Bleu (Les), 1961. - Normandie, Paris, Hachette, 1065 p.

Guide Diamant (Le), 1926. - Normandie, Paris, Hachette, 300 p.

Guide Le Routard, 1998-1999 - Normandie. Paris, Hachette, 382 p.

\section{Bibliographie}

Burnet (L.), 1963. - Villégiature et tourisme sur les côtes de France, Paris, Hachette, 484 p.

Bardet (G.), 1892. - Plages du Nord et de la Normandie : de Dunkerque au Mont-Saint-Michel, coll. « Des Guides Dentu », p. 120-160 [http://gallica.bnf.fr, dossier «naissance du tourisme »].

Ceriani (G.), Duhamel (Ph.), Knafou (R.), Stock (M.), 2005, « Le tourisme et la rencontre de l'autre. Voyage au pays des idées reçues », L'autre, Cliniques, cultures et sociétés, vol. 6, n 1, p. 71-82.

Clari (D.), 1977. - La façade littorale de Paris, le tourisme sur la côte normande, étude géographique. Paris, Ophrys, $383 \mathrm{p}$.

Duhamel (Ph.), 2000. - «Résidents secondaires et nouveaux résidents à Majorque », ESPACES, n 176, p. 33-37.

ÉQUIPE MIT, 2005. - Tourismes 2, moments de lieux, Paris, Belin, coll. Mappemonde, 345 p.

6. Nous reprenons ici les critères et les mots utilisés dans l'exposé de la typologie des lieux touristiques réalisé par notre équipe et dont la dernière publication remonte à 2003 (Stock coord.). 
—, 2002. - Tourismes 1, lieux communs, Paris, Belin, coll. Mappemonde, 320 p.

—, 2000. - « La mise en tourisme des lieux : un outil de diagnostic », Mappemonde, vol. 57, n 1, p. 2-6.

James (C.) Dr, 1867. - Guide pratique aux Eaux minérales et aux bains de Mer, Paris, Masson, p. 350-352 [http://gallica.bnf.fr, dossier «naissance du tourisme»].

JoAnne (A.), 1875. - Département de la Seine-Inférieure, Paris, Hachette, 53 p.

Knafou (R.), Bruston (M.), Deprest (F.), Duhamel (Ph.), Gay (J.-C.) et Sacareau (I.), 1997. - « Pour une approche géographique du tourisme », L'espace géographique, tome 26, n³ 3, p. 193-204.

Saint Fargeau (G. DE), 1842. - Guide pittoresque, portatif et complet du voyageur en France. Paris, Firmin Didot Frères, 814p. [http://gallica.bnf.fr, dossier "naissance du tourisme »].

Stock (M.) (coord.), Dehoorne (O.), Duhamel (Ph.), Gay (J.-C.), Lazzarotti (O.), Sacareau (I.) et Violier (Ph.), 2003. - Le tourisme. Acteurs, lieux et enjeux. Paris, Belin, coll. «Belin Sup », 301 p.

\section{Remerciements}

Tous mes remerciements à Pierre Wallon dont la rencontre fut précieuse et le discours sur les Petites Dalles riche d'enseignements. Ces quelques heures passées ensemble ont nourri ma réflexion, me permettant de mettre en perspective de nombreux matériaux, de mieux comprendre l'origine et le fonctionnement des communautés vacancières.

Cet article a été reçu le le 10 juin 2007 et définitivement accepté le 24 mars 2008. 